\title{
Amplitude Flux, Probability Flux, and Gauge Invariance in the Finite Volume Scheme for the Schrödinger Equation
}

\author{
D.F. Gordon, B. Hafizi, and A.S. Landsman ${ }^{\dagger}$ \\ Plasma Physics Division, Naval Research Laboratory, Washington D.C. 20375 \\ ${ }^{\dagger}$ ETH Zürich, Zürich, Switzerland
}

\begin{abstract}
The time dependent Schrödinger equation can be put in a probability conserving, gauge invariant form, on arbitrary structured grids via finite volume discretization. The gauge terms in the discrete system cancel with a portion of the amplitude flux to produce abbreviated flux functions. The resulting time translation operator is strictly unitary, and is compatible with an efficient operator splitting scheme that allows for multi-dimensional simulation with complex grid geometries. Moreover, the abbreviated amplitude flux is necessary to the construction of a conservative probability current. This construction turns out to be important when computing Bohmian trajectories in multi-dimensions. Bohmian trajectories are useful in the interpretation of quantum mechanical phenomena such as tunneling ionization, and provide a bridge between quantum and classical regimes.
\end{abstract}

Keywords: Schrödinger Equation, Finite Volume, Bohmian Trajectories

\section{Introduction}

In a previous article [1], we described an algorithm for solving the time dependent Schrödinger equation (TDSE) in the Coulomb gauge, which strictly conserves probability on any structured grid with orthogonal basis vectors. The algorithm is particularly useful for simulating the photoionization of atoms, but can be applied equally well to any system where a non-relativistic electron is exposed to an arbitrary electromagnetic potential. In order to interpret the results of such simulations, it is sometimes useful to consider 
Bohmian particle trajectories in multi-dimensions. Bohmian mechanics has been receiving increasing attention in recent years $[2,3,4]$, partly because of its close connection to the theory of weak measurement [5]. For instance, weak measurement was used to measure the average trajectory of a photon in a two-slit experiment, showing that the resultant trajectories were Bohmian [2]. In laser ionization, Bohmian trajectories were used to gain a better understanding of High Harmonic Generation [6], and to interpret attosecond electron dynamics in a laser-driven hydrogen molecular ion [7]. The computation of Bohmian trajectories, however, still remains a challenge, even in the simpler case of static potentials [8].

The scheme described in [1] is a natural candidate for computing Bohmian trajectories since it is based on a finite volume scheme similar to those used in computational hydrodynamics [9]. The finite volume method is constructed around the notion that the average value of some conserved quantity in a cell is updated by calculating the flux that carries that quantity through each cell wall. In the case of the TDSE, the conserved quantity is probability, and the flux that carries it is probability current. Computing an accurate probability current makes the computation of Bohmian trajectories relatively straightforward.

This paper clarifies the relationship between the conservative amplitude flux that is used to update the wavefunction, and the conservative probability flux that is useful for computing Bohmian trajectories, among other things. The central concept is that of an "abbreviated" amplitude flux, which is formed by canceling terms in the true flux functions with terms involving $\nabla \cdot \mathbf{A}$, where $\mathbf{A}$ is the vector potential. The abbreviated amplitude flux is double valued in the sense that its value in a cell wall depends on which of the two adjacent cells is being updated. This double-valued flux must be used to construct a single-valued, strictly conservative probability current. It is found that without this construction, catastrophic errors appear in the Bohmian trajectories derived from the current. The use of abbreviated amplitude fluxes has two additional benefits: it leads to a gauge invariant algorithm, and it guarantees strict unitarity in some cases where the former algorithm does not. 


\section{True and Abbreviated Amplitude Fluxes}

For the purpose of finite volume evaluation, it is convenient to put the TDSE in the form [1]

$$
\partial_{t} \psi+\nabla \cdot \mathbf{f}=\mathrm{i} \chi \psi+\frac{1}{2}(\nabla \cdot \mathbf{A}) \psi
$$

where $\mathbf{f}$ is the amplitude flux density, $\psi$ is the wavefunction, and $\chi=\Phi-$ $A^{2} / 2$, with $\Phi$ the scalar potential. Here, and in all that follows, atomic units (a.u.) are employed. The flux density is given by

$$
\mathbf{f}=\left(\mathbf{A}-\frac{\mathrm{i}}{2} \nabla\right) \psi
$$

Volume integration over cell $\Omega_{i j k}$, with volume $V_{i j k}$, leads to the discretized TDSE [1]

$$
\begin{array}{r}
V_{i j k}\left(\partial_{t}-\mathrm{i} \chi_{i j k}\right) \psi_{i j k}-G_{i j k}= \\
F_{i-\frac{1}{2}, j, k}^{\xi}-F_{i+\frac{1}{2}, j, k}^{\xi}+F_{i, j-\frac{1}{2}, k}^{\eta}-F_{i, j+\frac{1}{2}, k}^{\eta}+F_{i, j, k-\frac{1}{2}}^{\zeta}-F_{i, j, k+\frac{1}{2}}^{\zeta}
\end{array}
$$

where the $F$, annotated in various ways, are the flux functions, $(\xi, \eta, \zeta)$ are arbitrary coordinates, and

$$
G_{i j k}=\frac{1}{2} \int_{\Omega_{i j k}} d^{3} \mathbf{r} \psi \nabla \cdot \mathbf{A}
$$

The flux functions are

$$
\begin{aligned}
& F_{i-\frac{1}{2}, j, k}^{\xi}=\left[-\frac{\mathrm{i}}{2} \frac{\psi_{i, j, k}-\psi_{i-1, j, k}}{\Delta_{i-\frac{1}{2}, j, k}^{\xi}}+\frac{A_{i-\frac{1}{2}, j, k}^{\xi}}{2}\left(\psi_{i-1, j, k}+\psi_{i, j, k}\right)\right] S_{i-\frac{1}{2}, j, k}^{\xi} \\
& F_{i, j-\frac{1}{2}, k}^{\eta}=\left[-\frac{\mathrm{i}}{2} \frac{\psi_{i, j, k}-\psi_{i, j-1, k}}{\Delta_{i, j-\frac{1}{2}, k}^{\eta}}+\frac{A_{i, j-\frac{1}{2}, k}^{\eta}}{2}\left(\psi_{i, j-1, k}+\psi_{i, j, k}\right)\right] S_{i, j-\frac{1}{2}, k}^{\eta} \\
& F_{i, j, k-\frac{1}{2}}^{\zeta}=\left[-\frac{\mathrm{i}}{2} \frac{\psi_{i, j, k}-\psi_{i, j, k-1}}{\Delta_{i, j, k-\frac{1}{2}}^{\zeta}}+\frac{A_{i, j, k-\frac{1}{2}}^{\zeta}}{2}\left(\psi_{i, j, k-1}+\psi_{i, j, k}\right)\right] S_{i, j, k-\frac{1}{2}}^{\zeta}
\end{aligned}
$$

where half-integer indices refer to the midpoint between cells (i.e., the value in a cell wall), and vector components appear in the superscript. For example, 
$S_{i-\frac{1}{2}, j, k}^{\xi}$ is the area of the cell wall between cells $(i-1, j, k)$ and $(i, j, k)$, with $S_{i, j-\frac{1}{2}, k}^{\eta}$ and $S_{i, j, k-\frac{1}{2}}^{\zeta}$ defined similarly. $\Delta$ is the arc-length between cell centers, similarly annotated.

We define "abbreviated" flux functions by evaluating $\nabla \cdot \mathbf{A}$ and canceling terms. In the Coulomb gauge, $\nabla \cdot \mathbf{A}=0, G_{i j k}=0$, and the finite volume gauge condition reads

$$
\begin{aligned}
& \left(S_{i+\frac{1}{2}, j, k}^{\xi} \mathbf{A}_{i+\frac{1}{2}, j, k}-S_{i-\frac{1}{2}, j, k}^{\xi} \mathbf{A}_{i-\frac{1}{2}, j, k}\right) \cdot \mathbf{e}_{\xi} \\
+ & \left(S_{i, j+\frac{1}{2}, k}^{\eta} \mathbf{A}_{i, j+\frac{1}{2}, k}-S_{i, j-\frac{1}{2}, k}^{\eta} \mathbf{A}_{i, j-\frac{1}{2}, k}\right) \cdot \mathbf{e}_{\eta} \\
+ & \left(S_{i, j, k+\frac{1}{2}}^{\zeta} \mathbf{A}_{i, j, k+\frac{1}{2}}-S_{i, j, k-\frac{1}{2}}^{\zeta} \mathbf{A}_{i, j, k-\frac{1}{2}}\right) \cdot \mathbf{e}_{\zeta}=0
\end{aligned}
$$

If this is used to eliminate terms in Eq. (3), the abbreviated flux functions, particular to the cell in question, are

$$
\begin{aligned}
& F_{i-\frac{1}{2}, j, k}^{+}=\left[-\frac{\mathrm{i}}{2} \frac{\psi_{i, j, k}-\psi_{i-1, j, k}}{\Delta_{i-\frac{1}{2}, j, k}^{\xi}}+\frac{A_{i-\frac{1}{2}, j, k}^{\xi}}{2} \psi_{i-1, j, k}\right] S_{i-\frac{1}{2}, j, k}^{\xi} \\
& F_{i, j-\frac{1}{2}, k}^{+}=\left[-\frac{\mathrm{i}}{2} \frac{\psi_{i, j, k}-\psi_{i, j-1, k}}{\Delta_{i, j-\frac{1}{2}, k}^{\eta}}+\frac{A_{i, j-\frac{1}{2}, k}^{\eta}}{2} \psi_{i, j-1, k}\right] S_{i, j-\frac{1}{2}, k}^{\eta} \\
& F_{i, j, k-\frac{1}{2}}^{+}=\left[-\frac{\mathrm{i}}{2} \frac{\psi_{i, j, k}-\psi_{i, j, k-1}}{\Delta_{i, j, k-\frac{1}{2}}^{\zeta}}+\frac{A_{i, j, k-\frac{1}{2}}^{\zeta}}{2} \psi_{i, j, k-1}\right] S_{i, j, k-\frac{1}{2}}^{\zeta} \\
& F_{i+\frac{1}{2}, j, k}^{-}=\left[-\frac{\mathrm{i}}{2} \frac{\psi_{i+1, j, k}-\psi_{i, j, k}}{\Delta_{i+\frac{1}{2}, j, k}^{\xi}}+\frac{A_{i+\frac{1}{2}, j, k}^{\xi}}{2} \psi_{i+1, j, k}\right] S_{i+\frac{1}{2}, j, k}^{\xi} \\
& F_{i, j+\frac{1}{2}, k}^{-}=\left[-\frac{\mathrm{i}}{2} \frac{\psi_{i, j+1, k}-\psi_{i, j, k}}{\Delta_{i, j+\frac{1}{2}, k}^{\eta}}+\frac{A_{i, j+\frac{1}{2}, k}^{\eta}}{2} \psi_{i, j+1, k}\right] S_{i, j+\frac{1}{2}, k}^{\eta} \\
& F_{i, j, k+\frac{1}{2}}^{-}=\left[-\frac{\mathrm{i}}{2} \frac{\psi_{i, j, k+1}-\psi_{i, j, k}}{\Delta_{i, j, k+\frac{1}{2}}^{\zeta}}+\frac{A_{i, j, k+\frac{1}{2}}^{\zeta}}{2} \psi_{i, j, k+1}\right] S_{i, j, k+\frac{1}{2}}^{\zeta}
\end{aligned}
$$

Here, the \pm superscript on $F$ is used to indicate the direction in which the cell that is affected by the abbreviated flux lies. Due to the need for extensive 
annotations, the superscript indicating the vector component is suppressed. It can be deduced by noting which of the cell indices has the half-integral value.

It should be stressed that $F_{i-\frac{1}{2}, j, k}^{+} \neq F_{i-\frac{1}{2}, j, k}^{-}$, as can be seen by inspection. This says that the abbreviated flux takes two different values in each cell wall. It is shown below that this double valued amplitude flux can, and must, be used to form a single valued, conservative, probability flux.

\section{Gauge Invariance}

In an arbitrary gauge, a suitable differencing scheme for $G_{i j k}$ is needed. Application of the finite volume approach is not straightforward, because the integrand in the definition of $G_{i j k}$ is not a total divergence. However, if the variation of $\psi$ is negligible within a single cell, then $\psi$ may be taken out of the integral, and finite volume evaluation of $G_{i j k}$ gives

$$
\begin{array}{r}
G_{i j k}=\frac{1}{2} \psi_{i, j, k}\left(S_{i+\frac{1}{2}, j, k}^{\xi} \mathbf{A}_{i+\frac{1}{2}, j, k}-S_{i-\frac{1}{2}, j, k}^{\xi} \mathbf{A}_{i-\frac{1}{2}, j, k}\right) \cdot \mathbf{e}_{\xi} \\
+\left(S_{i, j+\frac{1}{2}, k}^{\eta} \mathbf{A}_{i, j+\frac{1}{2}, k}-S_{i, j-\frac{1}{2}, k}^{\eta} \mathbf{A}_{i, j-\frac{1}{2}, k}\right) \cdot \mathbf{e}_{\eta} \\
+\left(S_{i, j, k+\frac{1}{2}}^{\zeta} \mathbf{A}_{i, j, k+\frac{1}{2}}-S_{i, j, k-\frac{1}{2}}^{\zeta} \mathbf{A}_{i, j, k-\frac{1}{2}}\right) \cdot \mathbf{e}_{\zeta}
\end{array}
$$

Each term in $G_{i j k}$ then cancels a corresponding term in the flux functions, leading exactly to the abbreviated fluxes given above. Then, in any gauge, the discretized TDSE takes the form

$$
\begin{array}{r}
V_{i j k}\left(\partial_{t}-\mathrm{i} \chi_{i j k}\right) \psi_{i j k}= \\
F_{i-\frac{1}{2}, j, k}^{+}-F_{i+\frac{1}{2}, j, k}^{-}+F_{i, j-\frac{1}{2}, k}^{+}-F_{i, j+\frac{1}{2}, k}^{-}+F_{i, j, k-\frac{1}{2}}^{+}-F_{i, j, k+\frac{1}{2}}^{-}
\end{array}
$$

Although Eq. (16) is gauge invariant to within a discretization error, the Coulomb gauge has the appealing characteristic that finite volume discretization can be carried out without approximation.

\section{Semi-Implicit Advance and Operator Splitting}

As pointed out in [1], when the time differencing in Eq. (3) is carried out semi-implicitly, and the Coulomb gauge is employed, the discretized time advance operator,

$$
U=\frac{1-\frac{1}{2} \mathrm{i} H \Delta t}{1+\frac{1}{2} \mathrm{i} H \Delta t},
$$


is unitary to within a round-off error, so that probability is exactly conserved. This characteristic follows from the Hermiticity of the discretized Hamiltonian matrix, $H$. However, when a multi-dimensional advance is carried out by means of operator splitting, the situation is more subtle. In the operator splitting scheme, the overall time advance is carried out by advancing each one dimensional strip independently. If the true amplitude flux is used, this leads to the one dimensional Hamiltonian [1]

$$
\begin{gathered}
H_{i, i-1}=\frac{S_{i-\frac{1}{2}}}{2 \sqrt{V_{i} V_{i-1}}}\left(\frac{-1}{\Delta_{i-\frac{1}{2}}}+\mathrm{i} A_{i-\frac{1}{2}}\right) \\
H_{i, i}=\frac{S_{i-\frac{1}{2}}}{2 V_{i}}\left(\frac{1}{\Delta_{i-\frac{1}{2}}}+\mathrm{i} A_{i-\frac{1}{2}}\right)+\frac{S_{i+\frac{1}{2}}}{2 V_{i}}\left(\frac{1}{\Delta_{i+\frac{1}{2}}}-\mathrm{i} A_{i+\frac{1}{2}}\right)-\frac{\chi_{i}}{D} \\
H_{i-1, i}=\frac{S_{i-\frac{1}{2}}}{2 \sqrt{V_{i} V_{i-1}}}\left(\frac{-1}{\Delta_{i-\frac{1}{2}}}-\mathrm{i} A_{i-\frac{1}{2}}\right)
\end{gathered}
$$

where it is understood that $A$ and $\chi$ are evaluated half-way through the time step, and $D$ is the number of spatial dimensions. It was stated in [1] that this is "clearly Hermitian," which assumes

$$
S_{i-\frac{1}{2}} A_{i-\frac{1}{2}}-S_{i+\frac{1}{2}} A_{i+\frac{1}{2}}=0
$$

Actually, this condition does not hold in general, since the full gauge condition is the more complicated expression (8). Examples where (21) does hold include the most typical cases: Cartesian or cylindrical grids with the vector potential in the form of a plane wave. A simple example where it does not hold is a plane wave on a parabolic grid.

In order to develop a scheme which remains conservative even when operator splitting is used on a complex grid, replace Eq. (3) with Eq. (16). Although these two equations are equivalent mathematically, they have different characteristics with respect to operator splitting. In particular, when the abbreviated fluxes are used, the Hamiltonian used to update a one dimensional strip becomes

$$
H_{i, i-1}=\frac{S_{i-\frac{1}{2}}}{2 \sqrt{V_{i} V_{i-1}}}\left(\frac{-1}{\Delta_{i-\frac{1}{2}}}+\mathrm{i} A_{i-\frac{1}{2}}\right)
$$




$$
\begin{aligned}
H_{i, i} & =\frac{S_{i-\frac{1}{2}}}{2 V_{i} \Delta_{i-\frac{1}{2}}}+\frac{S_{i+\frac{1}{2}}}{2 V_{i} \Delta_{i+\frac{1}{2}}}-\frac{\chi_{i}}{D} \\
H_{i-1, i} & =\frac{S_{i-\frac{1}{2}}}{2 \sqrt{V_{i} V_{i-1}}}\left(\frac{-1}{\Delta_{i-\frac{1}{2}}}-\mathrm{i} A_{i-\frac{1}{2}}\right)
\end{aligned}
$$

which is clearly, and manifestly, Hermitian. Based on this observation, the abbreviated fluxes should be used to construct $U$. This change in algorithm may be viewed as inserting into the sequence of operations an operation that leaves the exact solution of (3) unchanged. There is, however, an effect on subsequent operations, since the operator splitting approach does not produce the exact solution of (3).

In summary, the use of the true flux is harmless in certain commonly occurring cases, but violates the unitarity of $U$ in cases of complex grid geometries or field configurations. Use of the abbreviated flux accounts for arbitrary gauge fixing, and gives a strictly unitary $U$ no matter the form of the grid or applied field. This generalization is required, e.g., to perform tunneling ionization simulations on a parabolic grid $^{1}$. It is also required for the construction of a conservative probability current, which in turn, is needed to calculate Bohmian trajectories.

\section{Construction of Probability Current}

For diagnostic purposes, one sometimes wants the probability current. The differential expression for the probability current density is

$$
\mathbf{j}=\frac{\mathrm{i}}{2}\left[\psi(\nabla-\mathrm{iA}) \psi^{*}-\psi^{*}(\nabla+\mathrm{iA}) \psi\right]
$$

If this is computed at a time level $n$ by taking centered differences of $\psi_{i j k}^{n}$, the resulting $\mathbf{j}$ has pathological characteristics. This is illustrated in Fig. 1, which shows the currents, so computed, for a hydrogen $1 s$ state. An accurate solution should give $\mathbf{j}=0$, but instead, a constant, non-negligible, pattern of currents appears. If these currents were used to predict the evolution of $|\psi|^{2}$, the stationary state would break-up within an atomic unit of time, which is clearly an unacceptable behavior. In contrast, the conservative currents

\footnotetext{
${ }^{1}$ This is not discussed further in the present paper, because we have not discovered any advantage of a parabolic grid over a cylindrical one.
} 

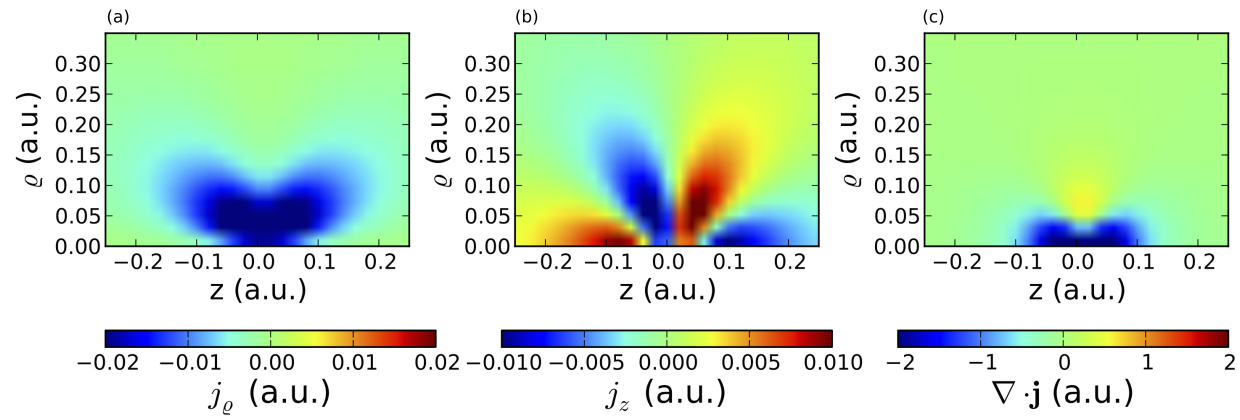

Figure 1: Spurious, constant currents associated with the $1 s$ state of hydrogen, resulting from centered differencing of Eq. (25). If the calculation is repeated using the conservative expression (28), the spurious currents are reduced by 2-3 orders of magnitude, and become oscillatory in time and space. Here, $\varrho$ is the cylindrical coordinate $\sqrt{x^{2}+y^{2}}$.

derived below preserve a stationary state for, at least, thousands of times longer. One reason for this is that the magnitude of the spurious current is reduced by two or three orders of magnitude. Another is that the spurious currents become oscillatory, so that their time-integrated effect is reduced.

To form a strictly conservative current, start with the semi-implicit finite volume advance for a one dimensional strip:

$$
2 V_{i} \frac{\left(\psi_{i}^{n+1}-\psi_{i}^{n}\right)}{\Delta t}=\mathrm{i} V_{i} \chi_{i}\left(\psi_{i}^{n}+\psi_{i}^{n+1}\right)+F_{i-\frac{1}{2}}^{+, n}+F_{i-\frac{1}{2}}^{+, n+1}-F_{i+\frac{1}{2}}^{-, n}-F_{i+\frac{1}{2}}^{-, n+1}
$$

Here, $n$ and $n+1$ are labels that refer to the quantity before and after the strip is updated (which in multi-dimensions is distinct from the time level index). Multiply by $\left(\psi_{i}^{n}+\psi_{i}^{n+1}\right)^{*}$ and add the complex conjugate (c.c.). This gives

$$
V_{i} \frac{\left|\psi_{i}^{n+1}\right|^{2}-\left|\psi_{i}^{n}\right|^{2}}{\Delta t}=j_{i-\frac{1}{2}} S_{i-\frac{1}{2}}-j_{i+\frac{1}{2}} S_{i+\frac{1}{2}}
$$

where

$$
j_{i-\frac{1}{2}}^{n+\frac{1}{2}}=\frac{1}{4}\left(\psi_{i}^{n *}+\psi_{i}^{n+1 *}\right)\left(f_{i-\frac{1}{2}}^{+, n}+f_{i-\frac{1}{2}}^{+, n+1}\right)+\text { c.c. }
$$

Here, $f_{i \pm \frac{1}{2}}=F_{i \pm \frac{1}{2}} / S_{i \pm \frac{1}{2}}$. Unlike the amplitude flux, the current density is single valued in a cell wall, i.e., $j_{i-\frac{1}{2}}^{+, n+\frac{1}{2}}=j_{i-\frac{1}{2}}^{-, n+\frac{1}{2}}=j_{i-\frac{1}{2}}^{n+\frac{1}{2}}$. This characteristic 
follows from the equality

$$
\left(\psi_{i}^{n *}+\psi_{i}^{n+1 *}\right)\left(f_{i-\frac{1}{2}}^{+, n}+f_{i-\frac{1}{2}}^{+, n+1}\right)+c . c .=\left(\psi_{i-1}^{n *}+\psi_{i-1}^{n+1 *}\right)\left(f_{i-\frac{1}{2}}^{-, n}+f_{i-\frac{1}{2}}^{-, n+1}\right)+c . c .
$$

which can be verified by direct expansion. The expression for the current density can be put in a form that more closely resembles the differential expression by defining the forward differencing operator $\hat{\delta}^{+} \psi_{i-1}=\left(\psi_{i}-\right.$ $\left.\psi_{i-1}\right) / \Delta_{i-\frac{1}{2}}$. Then,

$$
j_{i-\frac{1}{2}}^{n+\frac{1}{2}}=\frac{\mathrm{i}}{8} \sum_{l=n}^{n+1} \sum_{m=n}^{n+1}\left[\psi_{i}^{l}\left(\hat{\delta}^{+}-\mathrm{i} A_{i-\frac{1}{2}}^{n+\frac{1}{2}}\right) \psi_{i-1}^{m *}-\psi_{i}^{l *}\left(\hat{\delta}^{+}+\mathrm{i} A_{i-\frac{1}{2}}^{n+\frac{1}{2}}\right) \psi_{i-1}^{m}\right]
$$

It is interesting to note that a single valued probability current is arrived at only by making use of the abbreviated amplitude flux, which is double valued. If the single valued "true" amplitude flux is used, the probability current is double valued, and cannot be sensibly associated with any conservation law. As is well known in fundamental physics, gauge symmetry leads to charge conservation. Similarly, the gauge condition has to be invoked in order to form the conservative probability current (28).

\section{Bohmian Trajectories}

Knowing the current density, one can plot the Bohmian trajectories associated with an evolving wavefunction. These trajectories are defined by $d \mathbf{r} / d t=\mathbf{j} /|\psi|^{2}$. The Bohmian particles satisfy a Hamilton-Jacobi equation, with a quantum potential, that is exactly equivalent to the original Schrödinger equation. To compute the Bohmian trajectories numerically, particle-in-cell (PIC) techniques are employed. At the start of a step, the known quantities are $\psi^{n}, \mathbf{r}^{n}$, and $\mathbf{v}^{n-\frac{1}{2}}$, where $n$ is the time level index, $\mathbf{r}$ is the particle position, and $\mathbf{v}$ is the particle velocity. During the update of $\psi$, the current density is computed as described above, giving $\mathbf{j}^{n+\frac{1}{2}}$ and $\rho^{n+\frac{1}{2}}=\frac{1}{2}\left|\psi^{n}\right|^{2}+\frac{1}{2}\left|\psi^{n+1}\right|^{2}$. Then, $\rho^{n+\frac{1}{2}}$ and $\mathbf{j}^{n+\frac{1}{2}}$ are evaluated at $\mathbf{r}^{n+\frac{1}{2}}$ by second order interpolation from the grid. This requires the use of the approximation $\mathbf{r}^{n+\frac{1}{2}} \approx \mathbf{r}^{n}+\frac{1}{2} \mathbf{v}^{n-\frac{1}{2}} \Delta t$. Let the interpolated values be $\bar{\rho}$ and $\overline{\mathbf{j}}$. Then, the step is completed via $\mathbf{v}^{n+1 / 2}=\overline{\mathbf{j}} / \bar{\rho}$ and $\mathbf{r}^{n+1}=\mathbf{r}^{n}+\mathbf{v}^{n+\frac{1}{2}} \Delta t$.

As an example of Bohmian trajectories, consider a resonantly driven transition between two atomic states. The numerical atom has soft core potential

$$
\Phi=\frac{1}{\sqrt{r^{2}+\delta r^{2}}}
$$



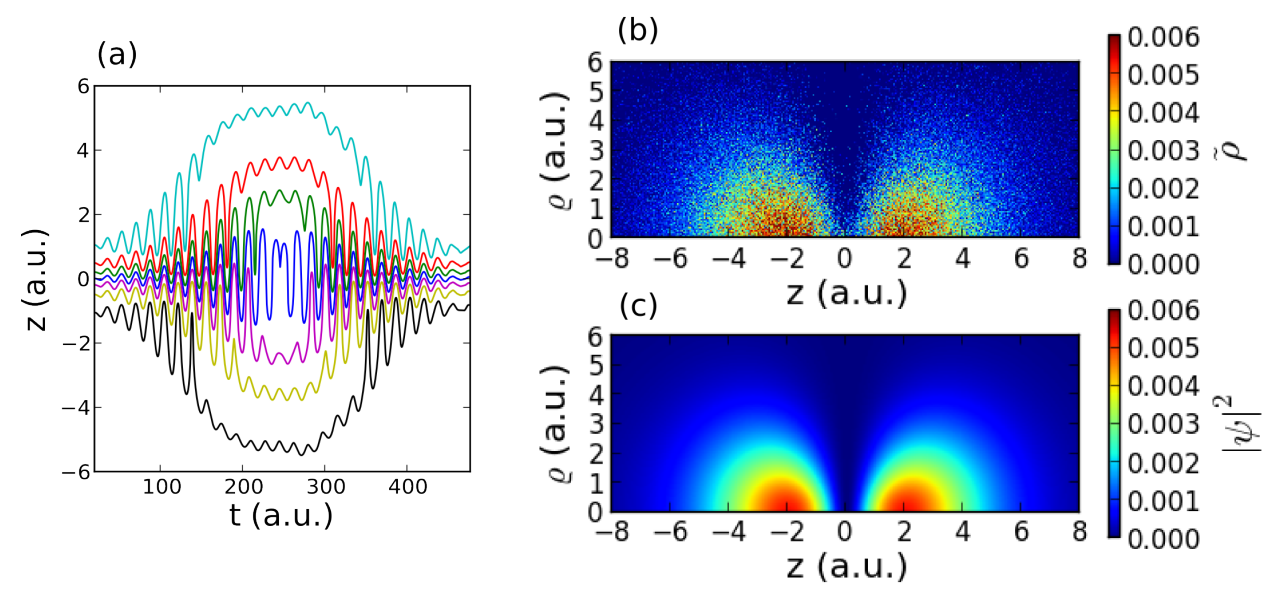

Figure 2: Simulation of Rabi oscillations between $1 s$ and $2 p$ states of hydrogen, with Bohmian trajectories computed using Eq. (28). (a) Bohmian trajectories during an interval $2 \pi / \omega_{R}$, where $\omega_{R}$ is the analytically determined Rabi frequency. (b) Density of Bohmian particles at $t=t_{0}+\pi / \omega_{R}$. (c) Expected density of Bohmian particles at $t=t_{0}+\pi / \omega_{R}$ in the limit of infinite particle number.

and is exposed to an applied field of the form

$$
\mathbf{A}=\frac{A_{0}}{2}\left[\cos \omega\left(t-t_{0}\right)-1\right] \Theta\left(t-t_{0}\right) \mathbf{e}_{z}
$$

Here, $\Theta$ is the Heaviside step function. For the simulation, an axisymmetric grid is used, with coordinates $\left\{\varrho=\left(x^{2}+y^{2}\right)^{1 / 2}, z\right\}$, cell sizes $\Delta \varrho=\Delta z=0.02$, and time step $\Delta t=0.005$. The soft core radius is $\delta r=0.04$. The two lowest energy levels are found numerically to be $E_{0}=-0.494$ and $E_{1}=-0.125$, with transition energy $\Delta E=0.369$. The initial state is formed by propagation in imaginary time (relaxation to ground) for an interval $t_{0}=20$. The radiation pulse has parameters $A_{0}=0.1$, and $\omega=\Delta E=0.369$. The Rabi frequency associated with these parameters is $\omega_{R}=0.0137$.

Fig. 2(a) shows several Bohmian trajectories computed based on Eq.(28). The particles are initialized on the $z$-axis, with positions chosen such that

$$
\left|\psi\left(t=0, \varrho=0, z_{i}\right)\right|^{2} \propto \begin{cases}\left|z_{i+1}-z_{i}\right|^{-1} & , \quad z_{i}>0 \\ \left|z_{i-1}-z_{i}\right|^{-1} & , \quad z_{i}<0\end{cases}
$$

where $i$ indexes the particles. In words, the density of particles is proportional to the probability density, a condition which is supposed to hold at 
all subsequent times. The initial particle distribution has a particle density consistent with the $1 s$ state. During the first half-Rabi period, the trajectories move outward to positions consistent with a $2 p$ state. After a full Rabi period, the particles return their initial positions in the $1 s$ state. It is important to note that if $\mathbf{j}$ were computed by centered differencing of Eq. (25), several trajectories would quickly fall toward the origin and remain there, contrary to the correct behavior.

To show the $2 p$ state more clearly, Fig. 2(b) shows the density, $\tilde{\rho}$, of a much larger statistical distribution of Bohmian particles, halfway through the Rabi oscillation. The initial distribution is loaded using a Monte-Carlo technique that gives a $1 s$ state in the limit of infinite particle number. As the particles move during the interaction, they arrive at the arrangement shown in Fig. 2(b). This can be compared with the exact $2 p$ state shown in Fig. 2(c). The granularity in Fig. 2(b) is due to the statistical nature of the particle loading.

To quantitatively evaluate the accuracy of the simulation, comparison is made with the analytical model of a two-level system, which predicts the level populations as a function of time. Determining the level populations based on the Bohmian trajectories is not straightforward, so instead, ordinary overlap integrals are carried out with respect to the wavefunction, $\psi(\mathbf{r}, t)$. Then, an error measure that compares the Bohmian density, $\tilde{\rho}$, with the density directly given by the wavefunction, $\rho=|\psi|^{2}$, is computed. A suitable error measure is

$$
\epsilon=1-\int d^{3} \mathbf{r} \sqrt{\rho \tilde{\rho}}
$$

which vanishes if the Bohmian density and $|\psi|^{2}$ are identical, and becomes unity if the Bohmian particles lie entirely outside the support of $|\psi|^{2}$. The calculated level populations ${ }^{2}$ are shown in Fig. 3(a), and the error measure for four different particle numbers is shown in Fig. 3(b). The plot in Fig. 3(a) agrees closely with the usual two-level analysis [10]. In the plot, the horizontal axis spans exactly the analytical Rabi period. The numerical Rabi period is seen to be nearly the same. We verified that the overall shape of the curves is faithfully reproduced. The numerical result has the added feature

\footnotetext{
${ }^{2}$ Before carrying out the overlap integrals, we put the wavefunction in the $\mathbf{d} \cdot \mathbf{E}$ gauge. Note also, that when comparing with analysis, the rotating wave approximation should not be used.
} 

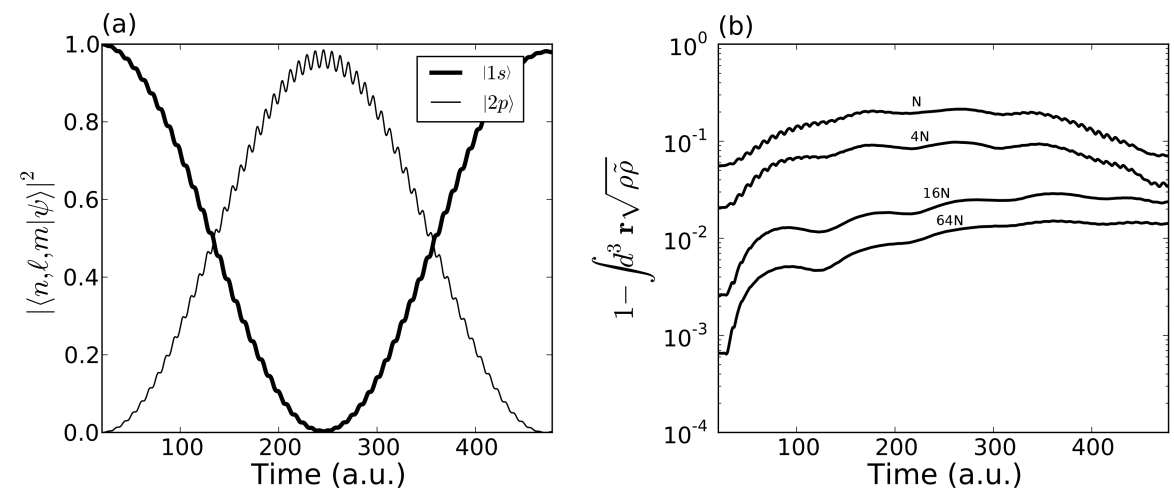

Figure 3: Data showing the accuracy of the simulation of Rabi oscillations between $1 \mathrm{~s}$ and $2 p$ states of hydrogen. (a) Populations of $1 s$ and $2 p$ states during the interaction. (b) Error in the Bohmian density with respect to the calculated wavefunction, for cases using $N, 4 N, 16 N$, and $64 N$ particles, where $N=10^{5}$.

that, after one Rabi period, the populations do not return exactly to their initial levels. This is because additional states, which are absent in the ideal two-level system, are excited in the numerical system.

Given that the wavefunction is calculated correctly, the error measure $\epsilon$ gives an indication of the accuracy of the Bohmian trajectories. There are two sources of error. The first is purely statistical, resulting from the Monte-Carlo particle loading technique. This error vanishes in the limit of large particle number. The second is due to discretization errors in the particle pusher. This error vanishes in the limit of small cell size and time step. Fig. 3(b) shows $\epsilon(t)$ for four different particle numbers. Each curve corresponds to a simulation with the grid parameters given above. The $\epsilon(t)$ curves result from a potentially complex interplay between statistical and discretization errors. For example, the curves for $N$ and $4 N$ particles have $\epsilon$ decreasing in time during the latter half of the simulation. This is not expected for a discretization error, but could be consistent with a statistical error. The curves with $16 N$ and $64 N$ particles are roughly monotone increasing, consistent with a discretization error. One can obtain a visual sense of the error by noting that Fig. 2(b) displays the $2 p$ state for $N=10^{5}$ particles, which gives $\epsilon \approx 0.2$ at the corresponding instant. In the case of $64 N$ particles, $\epsilon \approx 0.01$ at the same instant. 


\section{Conclusion}

The finite volume scheme for the time dependent Schrödinger equation can be put in a strictly unitary form that is compatible with operator splitting on complex structured grids. The approach makes use of gauge symmetry by canceling terms in the amplitude flux with terms involving the gauge condition. Using the resulting abbreviated amplitude flux, an exact probability conserving current density can be constructed. The current density, so constructed, is an essential element in the accurate, multi-dimensional, calculation of the Bohmian particle trajectories that are associated with an evolving wavefunction.

The algorithm described herein is useful for simulating a wide variety of physical processes, including tunneling ionization [11, 12, 13], high harmonic generation [14], terahertz radiation generation [15], and the nonlinear optical response of gases [16]. The ability to compute Bohmian trajectories in multi-dimensions brings interpretive insights that are not available in the conventional view. Furthermore, the Bohmian view naturally bridges the gap between the quantum and classical regimes, so that classical particle distributions can be readily determined in the appropriate spatio-temporal limits. Such classical distributions are useful in making contact with a class of experiments, in which electrons are observed in the classical region, very far from the atom.

\section{Acknowledgements}

This work is supported by the the Naval Research Laboratory 6.1 Base Program, and the U.S. Department of Energy (DOE) under Grant DE-AI0293ER40797. We appreciate useful discussions with M.H. Helle, A. Ting, J.R. Peñano, A. Zigler, J. Palastro, T. Rensink, and T. Antonsen, Jr.. Resources of the National Energy Research Scientific Computing Center (NERSC), and of the U.S. Department of Defense High Performance Computing and Modernization Program (HPCMP), were used in this work.

\section{References}

[1] D. Gordon, B. Hafizi, Time dependent schroedinger equation on arbitrary structured grids: application to photoionization, J. Comp. Phys. 231 (2012) 6349-6359. 
[2] S. Kocsis, B. Braverman, S. Ravets, M. Stevens, R. Mirin, L. Shalm, A. Steinberg, Observing the average trajectories of single photons in a two-slit interferometer, Science 332 (2011) 1170-1173.

[3] B. Braverman, C. Simon, Proposal to observe the nonlocality of Bohmian trajectories with entangled photons, Phys. Rev. Lett. 110 (2013) 060406-1-060406-5.

[4] H. Wiseman, Grounding Bohmian mechanics in weak values and bayesianism, New J. Phys. 9 (2007) 165-1-165-12.

[5] Y. Aharonov, D. Albert, L. Vaidman, How the result of a measurement of a component of the spin of a spin- $1 / 2$ particle can turn out to be 100 , Phys. Rev. Lett. 60 (1988) 1351-1354.

[6] J. Wu, B. Augstein, C. F. de Morisson Faria, Local dynamics in highorder harmonic generation using Bohmian trajectories, Phys. Rev. A 88 (2013) 023415-1-023415-7.

[7] N. Takemoto, A. Becker, Visualization and interpretation of attosecond electron dynamics in laser-driven hydrogen molecular ion using Bohmian trajectories, J. Chem. Phys. 134 (2011) 074309-1-074309-8.

[8] C. Lopreore, R. Wyatt, Quantum wave packet dynamics with trajectories, Phys. Rev. Lett. 82 (1999) 5190-5193.

[9] R. J. Leveque, Finite volume methods for hyperbolic problems, Cambridge University Press, 2002.

[10] P. Milonni, J. Eberly, Laser Physics, Wiley, Hoboken, NJ, 2010.

[11] R. Boge, C. Cirelli, A. Landsman, S. Heuser, A. Ludwig, J. Maurer, M. Weger, L. Gallmann, U. Keller, Probing nonadiabatic effects in strong-field tunnel ionization, Phys. Rev. Lett. 111 (2013) 103003-1103003-5.

[12] A. Landsman, C. Hofmann, A. Pfeiffer, C. Cirelli, U. Keller, A unified approach to probing Coulomb effects in tunnel ionization for any ellipticity of laser light, Phys. Rev. Lett. 111 (2013) 263001-1-263001-5. 
[13] A. Bandrauk, F. Fillion-Gourdeau, E. Lorin, Atoms and molecules in intense laser fields: gauge invariance of theory and models, J. Phys. B: At. Mol. Opt. Phys. 46 (2013) 153001-1-153001-33.

[14] D. Shafir, H. Soifer, B. Bruner, M. Dagan, Y. Mairesse, S. Patchkovskii, M. Ivanov, O. Smirnova, N. Dudovich, Resolving the time when an electron exits a tunnelling barrier, Nature 485 (2012) 343-346.

[15] N. Karpowicz, X.-C. Zhang, Coherent terahertz echo of tunnel ionization in gases, Phys. Rev. Lett. 102 (2009) 093001-1-093001-4.

[16] C. Köhler, R. Guichard, E. Lorin, S. Chelkowski, A. D. Bandrauk, L. Berge, S. Skupin, Saturation of the nonlinear refractive index in atomic gases, Phys. Rev. A 87 (2013) 043811-1-043811-9. 

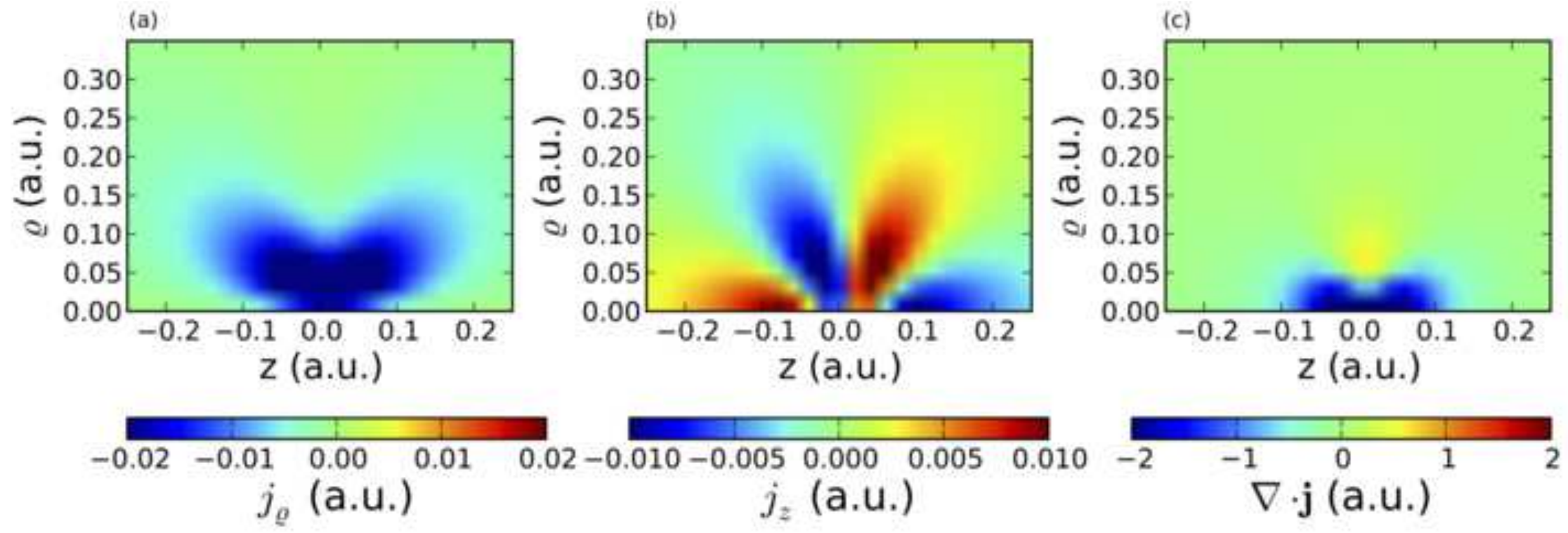

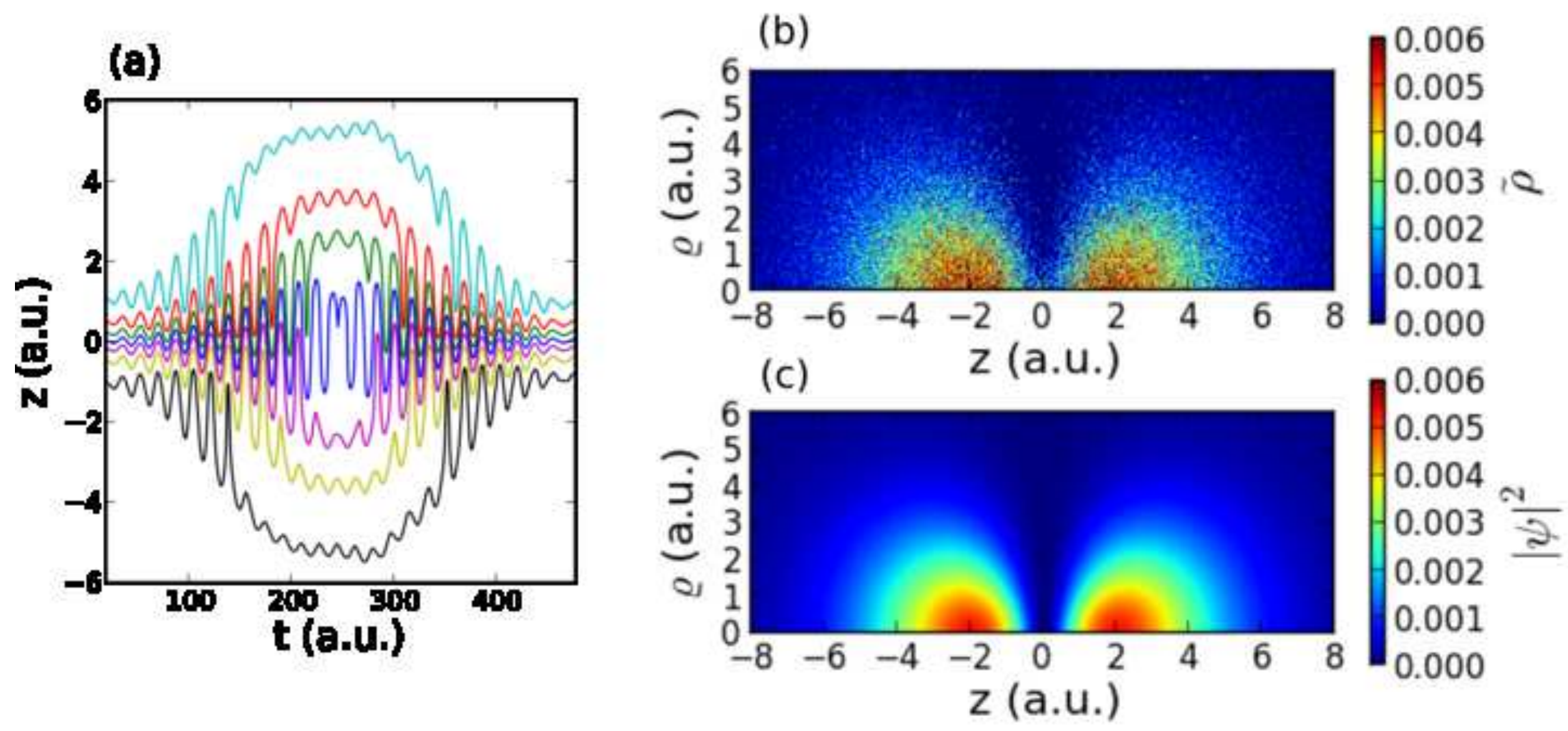

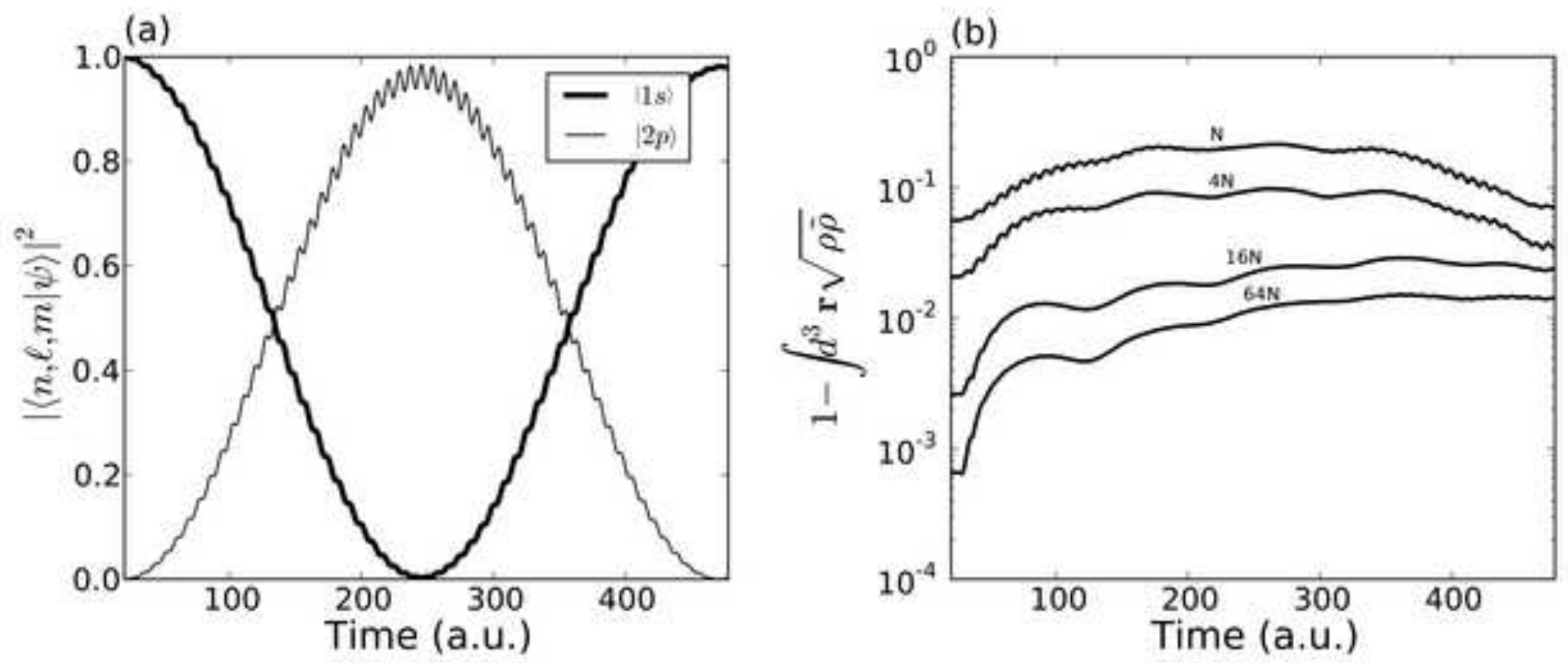